Offenbar war dieses Präparat nach Taddei's Methode durch Zusammenreiben von Quecksilber, Schwefel und reiner Schwefelleberlösung bereitet worden. Es ist möglich, dass sich hierbei eine kleine Quantität einer unlöslichen oder schwerlöslichen Verbindung von Schwefelkalium mit Schwefelquecksilber gebildet, und dass die Oxydation des Schwefelkaliums die des Schwefelquecksilbers eingeleitet hat.

\title{
Pharmaceutisch - chemische Notizen über Honig und Honigpräparate ;
}

O t t o Köhnke in Garding.

Wenn der Honig wohl zu den weniger wirksamen Heilmitteln gezählt werden kann, da der Hauptbestandtheil Krümelzucker ist, so ist doch sowohl nach älteren Schriftstellern, wie auch nach den neueren Erfahrungen nicht in Abrede zu stellen, dass derselbe dem leidenden Körper in manchen Fällen theils innerlich, theils äusserlich vortreffliche Dienste leistet.

In den Arzneischatz ist sowohl der rohe weisse und braune, als auch der gereinigte Honig aufgenommen; da letzterer indess unter vielen Umständen ein sehr variables Präparat werden muss, in sofern der rohe Honig nach der Oertlichkeit von weisser oder brauner Farbe an und für sich schon wesentlich verschiedene physische Eigenschaften besitzt und ohne Zweifel abweichende Bestandtheile enthält, da ferner durch die Aufbewahrung und die Art und Weise der Darstellung bedeutende Abweichungen entstehen, so erscheint es vorerst nicht ganz gleichgïltig, ob man weissen oder braunen Honig zur Darstellung des gereinigten und der sonstigen Präparate verwendet, anderntheils wünschenswerth, denselben nach einem überall gleichbleibenden einfach praktischen Verfahren zu reinigen, ohne, wie vorausgesetzt werden muss, den Honig in seinen urspruinglichen Bestandtheilen zu verändern, wodurch sich namentlich, abgesehen von den Eigenthümlichkeiten eines 
jeden rohen Honigs, ein mehr übereinstimmendes Präparat erzielen lässt.

Leber die Farbe, Aufbewahrung, die dabei vorkommenden Veränderungen und die Reinigung des Honigs wage ich cinige gesammelte Erfahrungen folgen zu lassen.

Um die ersterc Aufgabe in Erwägung zu ziehen, müssen einige, freilich nicht völlig genügende Grïnde, da die Wirkungen nicht speciell bekannt sind, angeführt werden, die dem Honige von weisslicher, blassgelblicher oder hochgelblicher Farbe, welchen Honig man gemeiniglich unter dem Namen $\mathrm{Mel}$ album versteht, zu $\mathrm{Mel}$ depuratum den Vorzug einräumen. Dor weisse Honig, kann man annehmen, ist grösstentheils immer derjenige, welcher von jungen Bienen gewonnen wird. Aeltere oder alte Bienen, deren Nahrung vorzugsweise die Flora des Buchweizens, der Heidekräuter oder der Zapfenbäume ist, machen eine Ausnahme, wesshalb man deren Honig mehr oder minder braun gefärbt findet, auch wohl mit dem Namen, ohne besondern Grund, Steinhonig belegt. Junge Bienen geben unter obigen Umständen, so oft sie mir vorgekommen, einen weisslichen, oder doch nur blassgelben Honig, gleichviel, ob derselbe ohne Wärme u. s. w., oder von den Rückständen durch Wärme und Auspressung gewonnen wurde; auch lassen zwei Bienenstöcke, welche neben einander stehen und worin in dem einen junge, in dem andern alte Bienen hausen, sowohl hinsichtlich der Farbe als auch des angenehmeren Geschmacks, sogleich den wesentlichen Unterschied erkennen. Mehr im Gegentheil zu dem Gesagten wird gemeiniglich angenommen, dass, nachdem der freiwillig aus den Honigwaben laufende Honig entfernt worden, der sodann aus den Zellen durch warme Behandlung und Auspressung gewonnene minder gut und braun gefärbt sei; dieses ist jedoch, wie ich glaube, irrthümlich und beruht, wenn nicht obiger gedachter Fall vorhanden, wo uberhaupt nur brauner Honig gewonnen wird, auf der Behandlung, welche man dabei befolgt.

Grosse Wärme und zu starke Auspressung werden, wie einleuchtend, unter allen Umständen einen sehr un- 
reinen und theilweise veränderten braunen Honig liefern, und hierauf scheint wohl vielfaltig die Farbe und Guite begründet. In manchen Gegenden, wo eine besondere Sorgfalt auf die Gewinnung des Honigs gelegt wird, die Rückstände ebenfalls erwärmt und ausgepresst werden, kennt man nur weissen oder weisslichen Honig, wozu allerdings die Flora beiträgt, umsichtige Behandlung aber immer ein nothwendiges Erforderniss bleibt. Abgesehen übrigens von den bereits angeführten Gründen, so besitzt der verschiedentlich braun gefärbte Honig, er mag gewonnen sein wo er will und wie er will, immer einen weniger angenehmen Geruch, als der weissliche, und in Bezug auf letztern einen stärker im Schlunde kratzenden Geschmack, wird weniger fest und hart, womit dann zugleich ein geringeres spec. Gew. und eine kürzere Haltbarkeit verbunden sind.

Verschiedene Proben frischen Honigs gaben folgende spec. Gewichte.

1) Hochgelber und blassgelblicher Honig von jungen Bienen, deren Nahrung vorzugsweise Buchweizenbliuthe war, erstarrte nach 3 und 4 Wochen zu einer krümlich schmierigen, jedoch sehr festen Masse; spec. Gew. 1,425-1,429. Honig von alten Bienen ebendaher erstarrte nach 4 und 6 Wochen zu einer mehr krümlich schmierigen Masse; spec. Gew. 1,415-1,422.

2) Heidehonig von blassgelber Farbe, härter als der vorhergehende; spec. Gew. 1,425-1,434. Hellbrauner von alten Bienen ebendaher; spec. Gew. 1,422-1,430.

3) Marschhonig von fast weisser Farbe, erhärtete nach 6 bis 8 Tagen und besass, am besten verglichen, die Härte, so wie uberhaupt das Ansehen des ausgeschmolzenen reinen Ochsentalgs; spec. Gew. 1,433-1,440.

Letzterer angeführte Honig ist unstreitig als eine der besten Sorten anzusehen, indem er alle Charaktere eines guten weissen Honigs im vorzüglichsten Grade in sich vereinigt; desshalb wird er denn auch im Handel neben dem zugleich erhaltenen Wachse entweder von blassgelblicher oder lebhaft citronengelber Farbe sehr gesucht. Nach dem spec. Gew. dieses Honigs, verglichen mit einer Krümel- 
zuckerlösung hinsichtlich des ersteren, so besteht derselbe fast aus reincm Krümelzucker. Die Nahrung der Bienen besteht vorzugsweise in der Flora des Rapses, der Feldbohnen (Vicia Faba) und des Klees, wesshalb der Honig nicht ganz allein in den Marschgegenden, sondern auch da, wie ich mich überzeugt, gewonnen wird, wo diese Feldgewächse gebauet werden.

Die Veränderung, welche der frische rohe Honig oft binnen kurzer Zeit erleidet, liegt grösstentheils in der Art und Weise der Aufbewahrung; bei einiger Sorgfalt kann man denselben je nach dem spec. Gew. 1, 2 bis 3 Jahre im fast ursprünglichen Zustande erhalten, wobei es indess erforderlich ist, den Honig aus guter Quelle zu beziehen und dem Bienenvater einige Anleitung zu geben. Man lässt zu diesem Zwecke kleine hölzerne Gebinde mit gut schliessendem Deckel anfertigen, kleine Gehinde desshalb, um je nach dem Verbrauche dieselben, sobald sie einmal angebrochen, binnen 3 höchstens \& Monaten entleeren zu kïnnen, und füllt dieselben sogleich mit dem aus den Honigwaben freiwillig oder auch aus den Rückständen durch gelinde Erwärmung, etwa +25 bis $30^{\circ} \mathrm{C}$., und schwache Auspressung gewonnenen Honig. Der Honig, nachdem er in einen trocknen Keller, oder an einen sonstigen trocknen, zugleich möglichst kühlen Ort gestellt worden, erhärtet nach dem spec. Gew. binnen 6 Tagen bis 4 und 6 Wochen, gleichviel ubrigens, wenn er erhärtet, man bedeckt die syrupsartige Masse allenthalben durch Andruicken mit genau nach dem Gefässe zugeschnittenem Wachspapier und verschliesst zu dem Ende sorgfältig mit dem Deckel. Irdene Töpfe zur Aufbewahrung des Honigs anzuwenden, ist verwerflich, indem sie meistens während des Erhärtens des Honigs durch die enitstehende Ausdehnung bersten, alsdann aber, in ein anderes Gefäss gebracht, tritt bei aller Umsicht, wohin gehört, dass man den Honig bei mässiger Wärme +25 bis $30^{\circ}$ völlig klar zerfliessen lässt und in das neue Standgefäss bringt, dennoch früher Zersetzung ein. Ich erlaube es mil demnach zu wiederholen, dass es eine unumgängliche durch die Praxis in Erfahrung gebrachte Bedingung ist, den fri- 
schen Honig sogleich in die hölzernen Gebinde zu bringen und darin krystallisiren zu lassen, wenn man denselben lange conserviren will.

Hinsichtlich der Aufbewahrung des Honigs ist, wie bereits angeführt, einige Sorgfalt nöthig, gegentheils erleidet derselbe über kurz eine bedeutende Veränderung; es bildet sich bekanntlich eine obenstehende Schicht Fluissigkeit, theilweise entstehend durch das Zerfliessen des Krümelzuckers an feuchter Luft, die darunter befindliche Masse wird schleimig, breiartig und pflanzt sich nach und nach durch das Ganze fort. Ein alter zerflossener Honig reagirt stark sauer und giebt, nachdem derselbe mit Kalkmilch gesättigt, wobei Ammoniak entwickelt wird, mit Hefe in die geistige Gährung gesetzt und weiter behandelt, grösstentheils milchsaure Kalkerde. Es unterliegt nach obigen Resultaten meines Bedünkens keinem Zweifel, dass der Honig in die schleimige Gährung geräth, zumal stickstoffhaltige Bestandtheile (Casein) sehr leicht in dem flockigen Bodensatze nachgewiesen werden können, welchen man erhält, wenn man mil Wasser verdünnten Honig mit Kali behandelt und das Filtrat genau mit Essigsäure sättigt. Belege zu einer völlig ähnlichen Bildung der schleimigen Gährung geben die neueren Entdeckungen von Liebig, Noellner, Pelouze und Gélis und andere Beobachtungen. Kommen zuckerhaltige Fliissigkeiten mit Casein bei einer Temperatur von +25 bis $30^{\circ}$ in Berührung, so werden anfänglich Milchsäure, später Buttersäure und letztere in vorherrschender Menge gebildet. Ein anderes Verhältniss findet nach meinen Beobachtungen statt, wenn man eine solche Mischung der Stubenwärme, etwa der Temperatur von +10 bis $15^{\circ}$ aussetzt; man erhält nach 8 bis 10 Wochen vorzugsweise Milchsäure, welche am zweckmässigsten während der Gährung an Kalkerde gebunden wird. Ferner verhält es sich ausnahmsweise ebenso mit Mellago Taraxaci. Der aus der frischen Pflanze, aus Leontodon Taraxacum gewonnene schwach sauer reagirende Saft, welcher nicht allein zuckerhaltige und stickstoffhaltige Bestandtheile, sondern auch, wie ich gefunden, fertig gebildete milchsaure 
Kalkerde enthält, liefert an einigen Orten immer, gleichviel mit welcher Abänderung dargestellt, einen Dicksaft, der binnen 6 bis 8 Wochen bei +5 bis $8^{\circ}$ Wärme in die schleimige Gährung übergeht; im Frübjahr bei +8 bis $12{ }^{\circ} \mathrm{Wärme}$ binnen 4 höchstens 6 Wochen. Nach Verlauf von einigen Monaten gesteht der Dicksaft zu einer festen harten Masse, reagirt sehr sauer und enthält eine grosse Menge, theils freie, theils an Ammonik gebundene Milchsäure.

Es ist bisher, wie ich glaube, allgemein vorausgesetzt, dass ein jeder Honig freie Säure enthalte, und dass sie unter andern Milchsäure sei; wenn nun gleich dieser Ansicht nach den bereits angefühten Thatsachen nichts entgegensteht, sondern vielmehr beim alten Honige bestätigt werden muss, so habe ich doch nicht unterlassen wollen zu bemerken, dass eine freie Säure nicht jedem frischen Honig eigenthiimlich ist. Der aus mehr denn 50 Bienenstöcken frisch abgelaufene fast farblose Marschhonig mit sorgfaltig bereitetem Lackmuspapier untersucht, ergab keine Reaction; dagegen zum Versuch einige Bienenstöcke zur Tödtung der Bienen mit brennendem Schwefel, wie es häufig geschieht, durchräuchert, gab, wie erklärlich, einen Spuren von Säure anzeigenden Honig. Proben von frischem Heidehonig u. s. w. hatte ich nach diesem Verfahren noch keine Gelegenheit auf freie Säure zu untersuchen.

Die Reinigung des Honigs bezweckt, die wachsartigen, wenig harzigen und die etwa mechanisch hineingefallenen Theile abzusondern; man wird demnach eine solche Methode anwenden müssen, wodurch jenes geschieht, ohne den Honig in seiner ursprüglichen Zusammensetzung zu beeinträchtigen. Kochender Honig oder auch Verdampfung grosser Mengen verdünnter Lösung, die beiden gewöhnlich angewandten Reinigungsmethoden, bedingen je nach der Länge der Zeit eine mehr oder minder grosse Zersetzung des Krimelzuckers und ohne Zweifel der übrigen Bestandtheile, wohin unter anderm auch die Verfluichtigung der ätherisch-aromatischen gehört. Die Veränderung des 
Zuckers und die dadurch herbeigeführte Menge unkrystallisirbaren Zuckers in Zuckersiedereien, ist genugsam bekannt.

Ein guter gereinigter Honig von 1,30 spec. Gew. muss bei einer Temperatur von +8 bis $10^{\circ}$ nach längerem Stehen uber $\frac{1}{y}$ Theil desselben an reinem krystallinischem Krümelzucker abscheiden, welches sonach ein gutes Zeichen, sowohl für die Güte des Präparates, als auch des angewandten rohen Honigs und der dabei befolgten Reinigungsmethode ist. Die Abscheidung des Krümelzuckers ist in mancher Hinsicht ein Uebelstand, jedoch, wie aus dem bereits Erwähnten hervorgeht, Bedingung eines guten Präparates, wesshalb es auffallend erscheinen muss, dass nach der Kenntniss von dem Hauptbestandtheile des Honigs, anderweitig darauf hingewiesen wird, dass diese Abscheidung des Zuckers durch die angegebene Behandlung zu beseitigen sei. Ein gereinigter Honig, in welchem sich kein Krümelzucker absetzt, muss jedenfalls als ein Präparat angesehen werden, welches unwerth ist, in den arzneilichen Gebrauch gezogen zu werden, indem entweder ein zersetzter oder mittelmässiger Honig angewendet, oder auch die Zersetzung durch langes Verdampfen oder Kochen, herbeigeführt wurde, welcher letztere Fall namentlich dasVerschwinden der fluichtigen Bestandtheile, so wie eine starke Farbung, die iibrigens nicht sowohl dem eigenthümlichen Farbstoffe, als vielmehr der Veränderung des Krümelzuckers zugeschrieben werden möchte, bedingt.

Unter allen Angaben zur Bereitung des gereinigten Honigs verdienen unstreitig diejenigen, nach welchen Kohle benutzt wird, sobald zugleich die nöthigen Vorsichtsmassregeln in Anwendung gebracht werden, den Vorzug, theils weil die trübenden Bestandtheile ohne Zersetzung des Honigs leicht getrennt werden, andernthcils die Ausbeute die grösstmöglichste ist, und abgesehen von der Farbe, auch der Riechstoff desselben, wie man entgegengesetzt vermuthen sollte, wenig leidet.

Die Darstellungsweisen der verschiedenen Honigpräparate, welche meines Bedünens am besten ihrem Zwecke entsprechen, erlaube ich mir nunmehr hier anzugeben. 


\section{Mel depuralum.}

$\mathrm{Zu}$ diesem Behufe bringt man in einen kupfernen gut verzinnten Kessel 40 Gewichtstheile festen Honig, 5 Gewichtstheile Wasser und 1 Gewichtstheil Thierkohle in erbsengrossen Stuicken und von dem feinen Pulver sorgfaltig getrennt, bedeckt den Kessel und legt einige glühende Kohlen unter, so, dass die Mischung eine Temperatur von +50 bis $60^{\circ} \mathrm{C}$. erreicht, worauf man diese Wärme 24. bis 36 Stunden lang unter bisweiligem Umrühren erhält, alsdann 1 bis 2 Minuten zum Sieden erhitzt und das Ganze in ein irdenes Gefäss giesst, welches einige Zeit etwa 6 bis 8 Tage lang, bei Seite gestellt wird. Sollte das spec. Gew. schwerer als 1,30 befunden werden, so setzl man gleich nach der Aufkochung genügend Wasser hinzu; bei einem Honig von niedrigem spec. Gew. wird dieser Fall nicht eintreten, sondern man wird vielmehr gezwungen sein, etwas weniger Wasser, als angegeben, zu nehmen. Nach Verlauf gedachter Zeit wird sich die Kohle auf den Boden des Gefässes fest abgelagert haben, nunmehr giesst man den schon fast klaren Honig auf ein dichtes, einfaches, oder auch zweifach zusammengelegtes wollenes Tuch mit der Vorsicht, dass der Bodensatz ungerührt bleibt. 40 bis 30 Pfund THonig von 4,30 spec. Gew. erhält man in einigen Stunden vollkommen rein und klar colirt.

Fehlt die zu diesem $Z$ wecke vorgeschriebene Thierkohle, so kann man auch, jedoch weniger empfehlungswerth, gute ausgewaschene Knochensch wärze (Ebur ustum nigrum) verwenden; der Honig muss in diesem Falle indess eine längere Zeil zum Asseizen bei Seite gestellt werden, oder man muss seine Zuflucht zu einigen Eiweissen nehmen, wozu auf circa 40 bis 50 Pfd. der Lösung 5 bis 6 gequirlte und mit dem Konig aufgekochte, ausreichen. Wurde brauner Honig angewandt, so erscheint der also erhaltene gereinigte von weingelber 2 arbe; weisser IIonig erfordert nur die Hälfte der angegebenen Koile, und erscheint alsdann farblos.

Der auf diese Weise erhaltene Honig besitzt den eigenthümlichen, etwas schwächeren Geruch des angewandten, 
den Geschmack des rohen und ist von tribenden, oder vielmehr wachsartigen Bestandtheilen vollkommen befreit. Die Reinheit des Honigs ergiebt sich durch eine sehr einfache Prüfung, die uiberhaupt auf den Eigenschaften der wachsartigen Körper beruht, indem man 1 bis 2 Drachmen des Präparates mit 4 bis 6 Unzen Wasser mischt. Der gut gereinigte Honig wird keine Flocken absetzen, im entgegengesetzten Falle werden sie in der Ruhe binnen wenigen Secunden erscheinen. Die zurückbleibende Kohle kann noch zu einigen technischen Zwecken verwandt werden. Der Verlust an Honig ist kaum in Rechnung zu bringen. Mel rosarum.

Die von der Landes-Pharmakopöe vorgeschriebene Menge Rosenblätter wird nach derselben mit Wasser infundirt. Man verdampft das colirte Infusum in einem Wasserbade bis zum dritten oder vierten Theile, erhitzt zum Sieden und setzt je nach der Menge 1, 2 bis 3 gequirlte Eiweisse hinzu, lässt einige Male aufwallen und colirt durch ein dichtes wollenes Tuch. Die erhaltene Fluissigkeit bringt man wiederum ins Wasserbad, verdunstet zur Consistenz eines dünnen Zuckersaftes, setzt diesen zu der Quantität des gereinigten Honigs, und lässt nunmehr aufkochen, worauf colirt wird. Man erhält einen Rosenhonig von durchsichtiger rothbräunlicher Farbe, der sich durch einen lieblichen Geruch und etwas adstringirenden, jedoch mehr angenehm süsslichen Geschmack auszeichnet.

Den Meerzwiebel - und Zeitlosensauerhonig bereitet man auf ähnliche Weise, indess die Eigenschaften des Eiweisses in Erwägung gezogen, so darf eine Klärung der dargestellten Essige mit demselben nicht gestattet werden, ist auch nicht nöthig, wenn man nur Sorge trägt, nachdem dieselben sich geklärt haben, durch weisses Papier zu filtriren, dieselben in einem Wasserbade zur Consistenz eines duinnflüssigen Zuckersaftes zu verdunsten, und nun mit dem gereinigten Honig gemischt und aufgekocht, durch ein wollenes Tuch zu coliren. Man erhält klare durchsichtige und je nach dem verwendeten Honig weingelbe oder schwach hellbräunliche Sauerhonige. 
pharm.-chem. Notizen über Honig. u. Honigpräparate. 49

\section{Oxymel simplex.}

Im Betreff des einfachen Sauerhonigs wage ich der schon anderweitig vorgeschlagenen Mischung von gereinigtem Honig mit concentrirtem Essig das Wort zu reden, indem ich glaube, dass sich nur auf diesem Wege ein mehr gleichbleibendes und unveränderliches Präparat erzielen lässt.

Von einigen Dispensatorien wird zur Bereitung Weinessig vorgeschrieben; es ist indess, wie ich voraussetzen darf, zu bekannt, in welchem Zustande derselbe in Norddeutschland und den nördlicheren Gegenden im Handel vorkommt, desshalb, abgesehen von der Essigsäure als Weinessig aus Wein oder Weinbeeren bereitet, gewiss ein sehr problematisches Heilmittel. Nimmt man nun die gewöhnlichen Verhältnisse von 2 Honig und 1 Essig an, so kommen auf 2 Pfd. à 12 Unzen gereinigten Honig, 1 Pfd. oder 12 Unzen Essig, wovon 2 Unzen 1 Drachme trocknes kohlensaures Kali neutralisiren; es werden mithin erfordert, um diesen Verhältnissen zu entsprechen, auf 2 Pfd. Honig $1 \frac{1}{2}$ Unzen Essigsäure, wovon 2 Drachmen 1 Drachme kohlensaures Kali neutralisiren, oder die ein spec. Gew. von 1,055 bis 1,060 besitzt. Sorgfältig angestellie Versuche haben indess ergeben, dass bei mehr oder minder starker Verdampfung der obigen Mischung mit verdünntem Essig zwischen $2 \frac{1}{2}$ bis $3 \frac{1}{2}$ Drachmen nach der Rechnung an concentrirter Essigsüure entweichen. Diesemnach wird sich also ein sehr gutes durchschnittliches Verhältniss gestalten, wenn man 3 Drachmen der gedachten concentrirten Essigsäure mit 8 Unzen gereinigtem Honig mischt. Dass man da, wo von der Landes - Pharmakopöe reiner roher Essig zu diesem Präparate vorgeschrieben ist, diese Verhältnisse anwenden darf, bedarf wohl keiner weiteren Erörterung. Je nach der Farbe des gereinigten Honigs erhält man einen farblosen oder weingelben Sauerhonig, von sehr angenehm säuerlichem Geruch und Geschmack. Oxymel aeruginis.

Es ist erwiesen, dass, wenn man eine neutrale essig saure Kupferoxydlösung mit einer zuckerhaltigen FlüssigArch, d. Pharm. XCIV. Bds. 1. Hft. 
keit kocht, oder nur anhaltend erwärmt, das Kupferoxyd zersetzt und grösstentheils zu Kupferoxydul reducirt, abgeschieden wird ${ }^{*}$ ).

Am zweckmässigsten möchte es demnach sein, 1 Gewichtstheil sehr fein zerriebenes neutrales essigsaures Kupferoxyd in $12-13$ Gewichtstheile gereinigten Honigs zu schuitten, die Mischung ohne Anwendung von Wärme unter bisweiligem Umschütteln einige Tage, sodann noch einige Tage zum Absetzen des ungelösten Salzes hinzustellen, worauf das mit möglichst vielem Kupferoxyd beladene Präparat klar von dem Bodensatze abzugiessen ist. Man erhält einen durchsichtigen bläulichgrünen Grünspanhonig, der, obgleich er schon nach einigen Wochen Kupferoxydul ausscheidet, selbst nach einem halben Jahre, noch aufgelöstes essigsaures Kupferoxyd in reichlicher Menge enthält; man wird übrigens auch in diesem Falle wohlthun, um ein möglichst gleichförmiges Präparat zu besitzen, die Mischung nur auf einige Monate vorräthig zu halten**).

*) Auf das Verhalten der Kupfersalze zu Zuckerlösungen beruht theilweise der vortheilhafte Gebrauch blank geschewerter kupferner Gefässe zur Aufkochung von Fruchtsäften. Die Fruchtsäfte, so wie auch der Veilchensyrup behalten, abgesehen von porcellanenen, nur in diesen Gefässen gekocht, ihre ursprüngliche lebhafte Farbe.

K.

**) Das auf gewöhnliche Weise bereitete Oxymel Aeruginis enthält auch nicht eine Spur von Kupfer aufgelöst, vornehmlich wenn das Präparat schon alt geworden war. Den braunen Bodensatz habe ich in kalter verdünnter Salzsäure meistens auflöslich, seltener unauflöslich gefunden. Derselbe ist daher gewöhnlich Kupferoxydul, und nur zuweilen fein zertheiltes Kupfer. H. W r. 